JOURNAL DE PHYSIQUE IV

Colloque C4, supplément au Journal de Physique III, Vol. 1, novembre 1991

C4-109

\title{
INDUSTRIAL PROCESSING OF COPPER BASED SHAPE MEMORY ALLOYS
}

\author{
G. CLÉMENT, P. NAUDOT and J-M. WELTER* \\ TREFIMETAUX, Centre de Recherche, BP. 11, F-60590 SERIFONTAINE, France \\ ${ }^{*}$ TREFIMETAUX, 11 bis rue de l'Hôtel de Ville, F-92400 Courbevoie, France
}

\begin{abstract}
In this paper, the production of SMA at TREFIMETAUX is described as well as the progressive change in the production of CuZnAl SMA to CuAlNi. We analyse the statistical dispersion of the transformation temperature of our hot extruded products and we precise the progresses obtained for three years. Remarks on hot transformation and heat treatments of CuAlNi at industrial scale complete this analysis.
\end{abstract}

The production of copper based shape Memory Alloy (SMA) began at TREFIMETAUX in 1985 for the zero insertion force connectors market: that was the CuZnAl with 4 wt\% Aluminium and transformation temperatures of about $-90^{\circ} \mathrm{C}$. He selected $4 \%$ to allow an hot and cold metallurgical transformation and to obtain small bars cross-sections : $4 \mathrm{~mm}^{2}$. In those years, the mastery of the transformation temperature was dependent on the composition as defined by the well known formula : Ms $=f$ (wt\% $\mathrm{Zn}$ ), and chemically analysed on final product; the accuracy of the temperature increased with new meltings.

The low limits of this alloy concerning ageing and fatigue have led us to develop simultaneously the CuZnAlNi alloy with 1 wt\% Ni; it is used now in some important applications.

The SMA market developed afterwards into higher transformation and usage temperatures : up to $200^{\circ} \mathrm{C}$ and sometimes beyond. Neither the two brass alloys nor the NiTi could fit this need; we had to introduce an other alloy : the CuAlNi with 4 wto Ni. The greater part of our research and industrial development during these last years was devoted to this alloy; it represents today our main product.

The pilot production made during the years 1985-1988 progressively evolved into the actual industrial productions on the following basis:

- increase of unitary molten and cast quantities, from 10 or $50 \mathrm{~kg}$ to 150 or $1000 \mathrm{~kg}$; units weighting about $3200 \mathrm{~kg}$ have been realised. 
- implementation and extension of elaboration and quality control principles applied in our brass, bronze and copper foundries to the elaboration of SMA, so that we can transfer the SMA production to our plants as soon as the quantities justify such an operation. So, an alloy can only be cast if its chemical composition has been analysed and found consistent with the customer specification; this rule suppose that one can adjust the liquid metal composition in the furnace (see fig. 1).

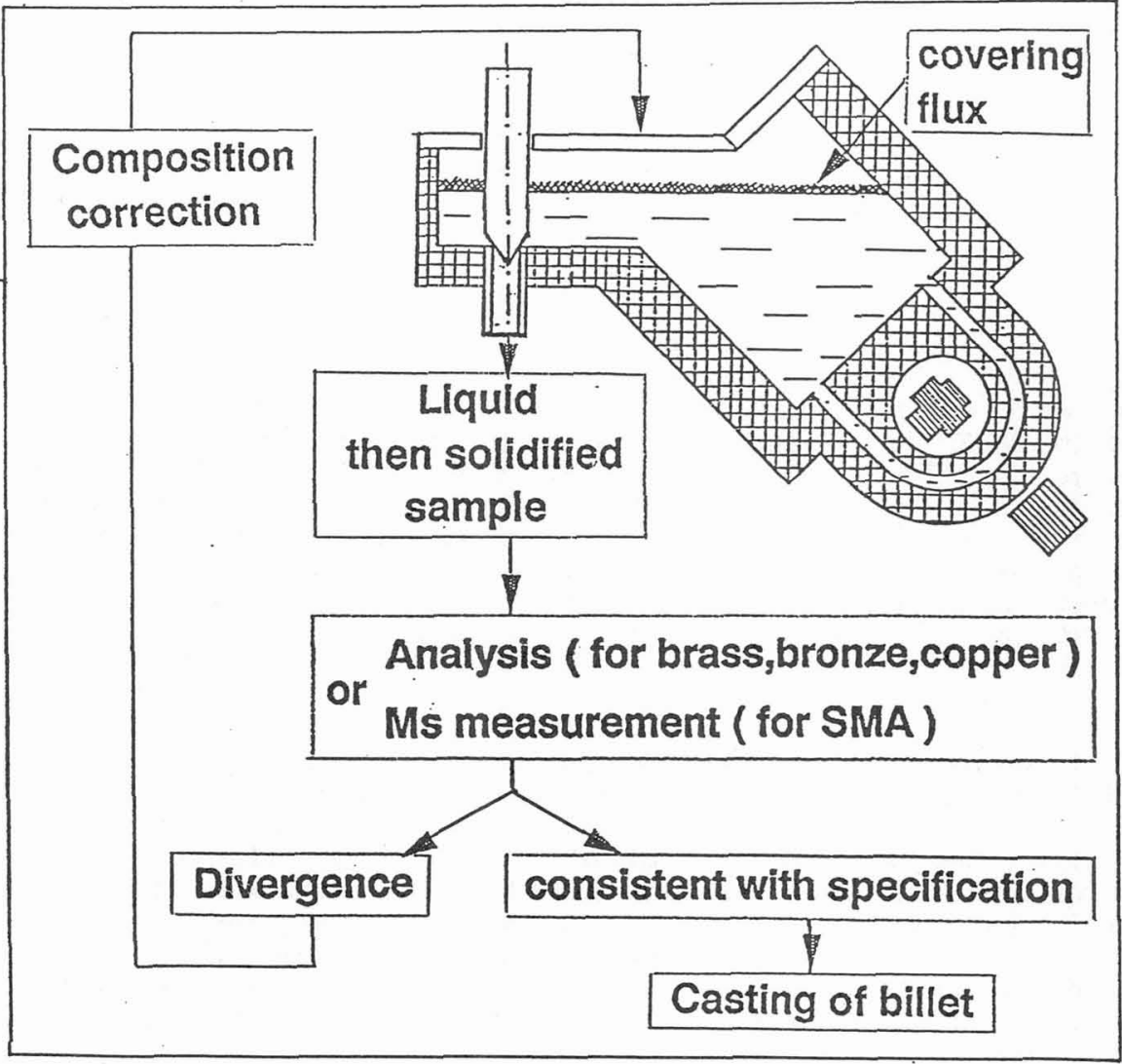

Fig.1 Principle of composition adjustment

It is more difficult to apply this principle to the SMA than to the other alloys :

* the accuracy of the chemical determination of zinc (0.16) has the same order of magnitude than the range of allowed composition of a SMA; but it represents only $10 \%$ of the allowed range of $a$ brass for forging and machining composition; the chemical analysis therefore is of no interest for a SMA in the melting furnace; then it is better to check directly the useful property for the customer : the transformation temperature; 
* nevertheless measuring the transformation temperature requires much more time than a spectro-chemical analysis. In particular during about one hour, we have to make the betatization and ageing treatment of a representative sample of molten alloy; but during this time, zinc evaporates from CuZnAl and consequently the sample transformation temperature is no longer representive of the molten alloy.

To avoid zinc and aluminium evaporation, our research led to a new covering flux, continuously lying on the molten alloy and absolutely blocking zinc and aluminium evaporation during the whole operation of controled elaboration of SMA.

In this way, we are sure that the transformation temperature mean value of cast billets or slabs matches our specifications; this conformity remains after the first hot transformation of the alloy.

Nevertheless this guarantee of the mean value for the transformation temperature is not sufficient for customers; we have now to examine the production according to its dispersion; the larger the unitary elaborated quantity, the more important this aspect is (see fig.2).

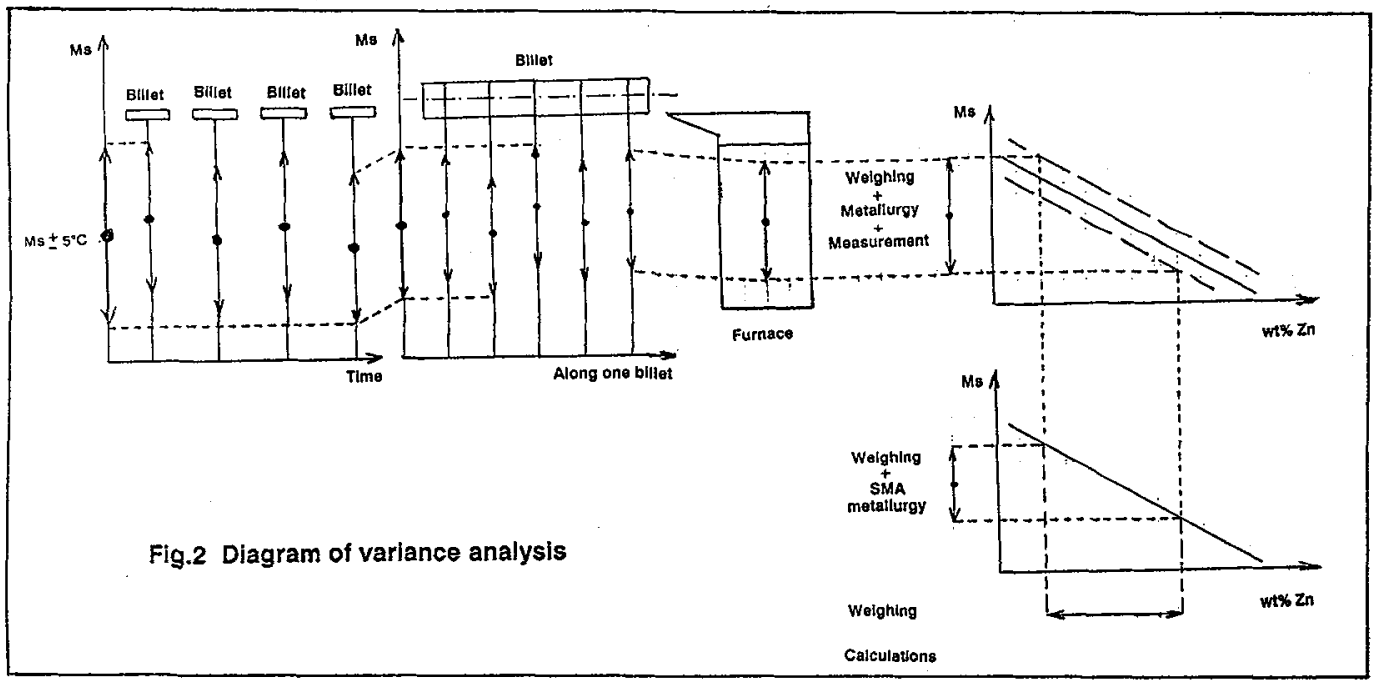

Between the molten and perfectly homogeneous alloy in the furnace and the solidified billet, there are many causes of scattering: the metal flowing from furnace to tundish, the progressive solidification during semi-continuous casting, the sampling and the measurement itself.

Considering a repetitive production, we have to distinguish two kinds of statistical dispersion for the SMA characteristics :

- the intra-unit dispersion, along a several meter long billet, for instance;

- the inter-unit dispersion, formed by variations of the units means. 
Here are our results in 1989 :

Sampling comes from four CuAlNi billets out of eight castings for which we aimed at the same transformation temperature Ms of $156^{\circ} \mathrm{C}$; in each billet, 28 blocks were cut at regular intervals; each block was hot extruded into a $3 \mathrm{~mm}$ diameter wire; this wire was treated at $850^{\circ} \mathrm{C}$ for 10 minutes, then quenched in water and aged at $220^{\circ} \mathrm{C}$ for 1 hour; the Ms temperatures were measured by the resistivity method according to the NFA 51-080 AFNOR standard "Alliages à mémoire de forme (AMF). Vocabulaire et mesure"; the measures taken in account are the ones obtained during the second martensitic transformation cycle after betatization.

Our results are summerized below:

\begin{tabular}{|l|c|c|c|c|}
\hline DATE & $15 / 03 / 89$ & $20 / 03 / 89$ & $31 / 07 / 89$ & $23 / 11 / 89$ \\
\hline number of samples & 28 & 28 & 28 & 28 \\
\hline Mean values Ms $\left({ }^{\circ} \mathrm{C}\right)$ & 155 & 158 & 157 & 154 \\
\hline Std-dev intra Ms & 4.7 & 3.0 & 2.9 & 3.4 \\
\hline Total Std-dev Ms & \multicolumn{3}{|c|}{3.9} \\
\hline $\begin{array}{l}\text { Repetability of } \\
\text { measured values } \\
\text { Std-dev Ms }\end{array}$ & 2.0 \\
\hline
\end{tabular}

The equation given by variance analysis of Ms temperatures is:

Total var = inter-unit var + intra-unit var + repetability var or numerically:

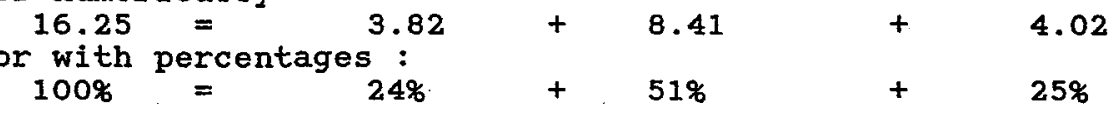

- The mean value of Ms, equal to $156^{\circ} \mathrm{C}$ matches exactly the one aimed at for this product.

- Onto the whole production, the Ms temperature varied at $95 \%$ in the range of : $156+/-7.8^{\circ} \mathrm{C}$.

To guarantee to our customers a dispersion of $t /-5^{\circ} \mathrm{C}$ on the delivered products, we had to eliminate an important part of them by sorting the production.

- The inter-unit variance has the same order of magnitude than the repetability of measures : thanks to a severe control of many elaboration parameters applying the statistical Process Control (SPC), we can reproduce, month after month, a given quality of SMA.

- The intra-unit variance which is the sum of variances due to sampling and betatization treatment is important; it is what we try to reduce as a priority. 
For units cast in 1990 , the whole production is in the range

for units cast in 1991 :

$$
157+/-6.6^{\circ} \mathrm{C}
$$

$155+/-5.7^{\circ} \mathrm{C}$.

our aim is to continue to reduce this range, well below $5^{\circ} \mathrm{C}$ in order to avoid batch selection.

In the first part of this talk, we have just described the foundry activity and billet quality; the next one is related to the SMA transformation.

As a SMA manufacturer, we must take at least the two further constraints into account :

- the one compelled by our customers : that is the diversity of final product shapes,

- the other by the metallurgy : the SMA's metallurgy is one of the most complicated among the copper based alloys.

The vast field of potential SMA applications, a very small part of which being explored until now, leads to create a new shape for each new final product. A large set of machines and tools able to generate these shapes are available at TREFIMETAUX and we can create new tools for new shapes.

Nevertheless, for economical reasons, we try to standardize shapes and temperatures; this standardization has recently known some success, giving evidence that customers are willing to accept our point of view.

The goal of development engineer is not to limit his own creativity by reducing the variety of shapes, but his job is to channel the potential customers' ideas toward existing products and technological solutions he believes relevant.

The SMA metallurgy always implies one first mechanical transformation at high temperature; the extrusion presses are very useful machines which fit the current need for small and variable quantities : with powers between 600 and 3200 tons, they allow us to draw circular solid and hollow sections and various flat bars useful for the SMA applications.

The metallurgy of the CuAlNi alloy set us specific problems : it is possible to reduce its section only at high temperature; so we have to generate the final shape in these conditions, preferabily with the extrusion press.

As this alloy is able to release high energies by memory effect, it is not necessary to use large cross-sections to develop an important mechanical work; we always try to get the smallest sections (up to $3 \mathrm{~mm}^{2}$ ) using the highest specific extrusion pressures (more than $100 \mathrm{daN} / \mathrm{mm}^{2}$ ) and the multiple flow extrusion technique : up to 8 identical $3 \mathrm{~mm}^{2}$ flat bars; even well mastered this technology has its own limits : creep strength of the die material, dimensional ranges of the shapes (see fig.3).... 


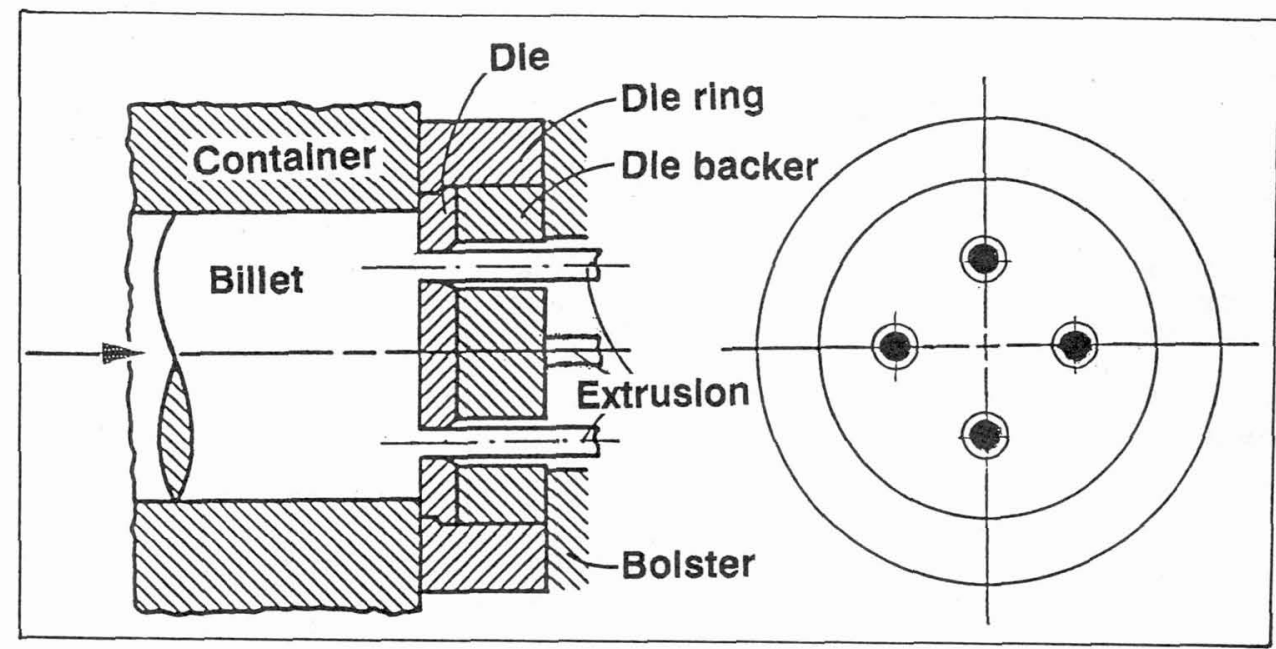

Fig.3 Shematic of a flat die for extrusion of small round bars

To reach smaller cross sections, we have to make use of complementary hot transformation technologies : hot rolling, hot hammering, hot wire drawing and so on (see fig.4).

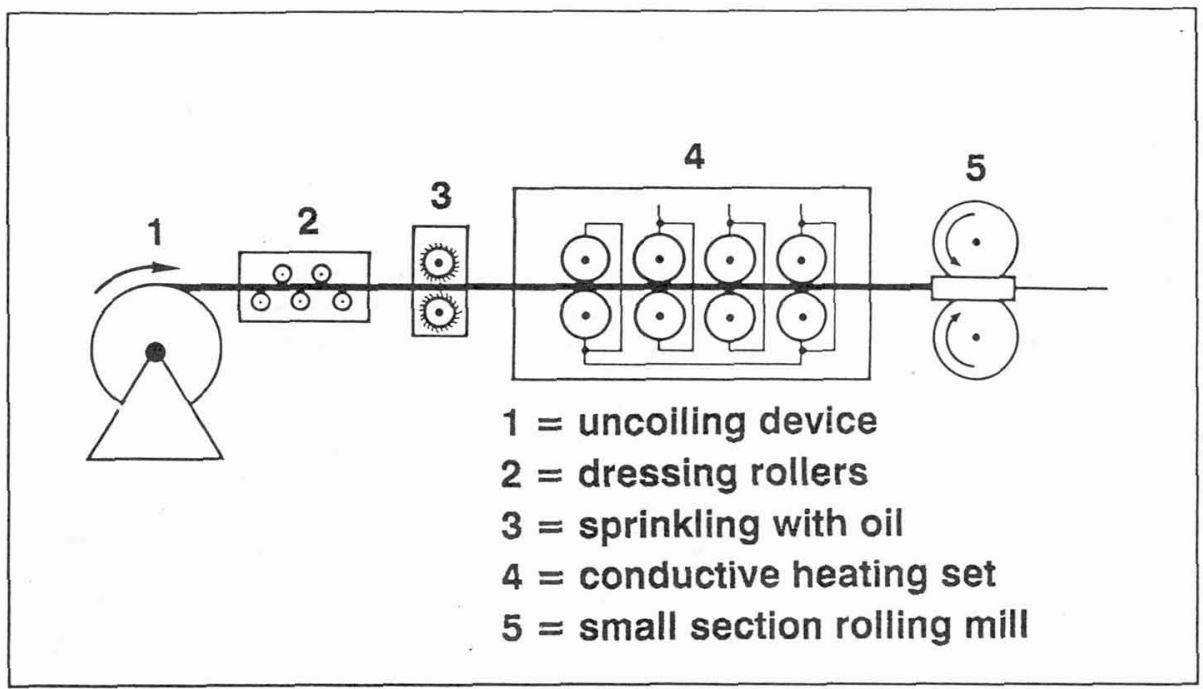

Fig.4 Principle of wire hot rolling mill 
Two main problems must be solved : wire heating and lubrication; heating by Joule effect with threephase current is suitable but the current supplies damage the lubricant film in front of the die; therefore, inductive heating is better, even if it requires powerful and expensive current generators, the yield of which decreases with the wire diameter; but this type of heating is compatible with an efficient lubrication.

Before building such machines, we have had to study thoroughly the CuAlNi ability to hot transformation, using tensile and torsional tests: the results analysis and their modelisation based on physical criteria allow us to optimize temperature conditions, alloy strain rate and reduction ratio (see fig.5).

Now it is possible to reduce the diameter of a CuAlNi wire down to $0.8 \mathrm{~mm}$.

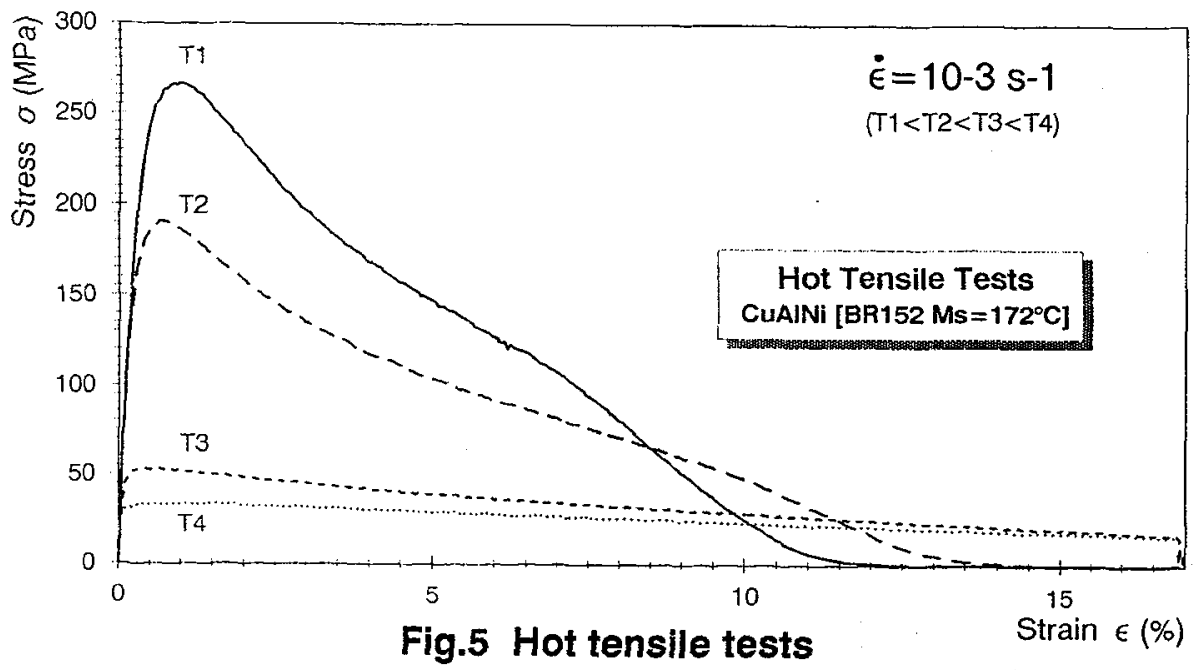

The SMA's heat treatments are critical operations in the production program :

- they can modify the transformation temperature,

- they control the grain size of the final product,

- they allow to optimize the fabrication,

- they confer the martensitic transformation the right qualities.

* the annealing treatments are appropriate to CuZnAl and CuznAlNi which accept a limited cold working ratio, about $15 \%$ between two heat treatments. The CuZnAl annealing at $500^{\circ} \mathrm{C}$ for 10 minutes is adequate to avoid grain growth, provided that the alloy contains grain refiner.

The annealing treatments must be made in oxidizing atmosphere; otherwise de-zincification and an increase of the transformation temperature appear. For a good reproductibility of Ms, it is necessary to overlook the cold working ratio and the number of intermediate annealings. 
* The heat treatments for memory effect (betatization) presents some difficulties, especialy passing from laboratory treatments on unitary devices to treatment of large series. After calculation of the high temperature shape, we have to put physically the device into this shape; for instance, it is easy to increase the diameter of a closed ring, but it is a non trivial problem to increase regularly the diameter of an open ring so that the memory effect could be used in the whole volume of the ring.

Then, this imposed shape has to be maintained during heating at $850^{\circ} \mathrm{C}$ for several minutes; it is well known that an SMA is very soft at this temperature. Therefore, we have to constrain it into a fixture which allows nevertheless the thermal expansion between room temperature and $850^{\circ} \mathrm{C}$; but this fixture protects and even reheats the device during quenching in cold or boiling water : the quench may be ineffective; this would be disastrous because this operation specifies the high temperature shape and gives the memory effect. The fixture solution found for one individual device is not necessary suitable for the treatment of series of devices.

In short, producing SMA is a modern and complete business :

- it is a modern business because it supposes :

* much specialized knowledge in SMA metallurgy,

* a vast know-how inherited from a long tradition in

copper based materials.

* important technical means in foundry and transformation used in the production of commodities,

* new means resulting from technological research, well adapted to production of specialities,

- it is a complete business because working from foundry elaboration to semi-final or final products, thus achieving the essential feedback which generates progress. 